\title{
ATIVIDADE ANTIOXIDANTE DO EXTRATO DE SEMENTES DE LIMÃO (Citrus limon) ADICIONADO AO ÓLEO DE SOJA EM TESTE DE ESTOCAGEM ACELERADA
}

\author{
Débora Maria Moreno Luzia e Neuza Jorge* \\ Departamento de Engenharia e Tecnologia de Alimentos, Universidade Estadual Paulista, Rua Cristóvão Colombo, 2265, \\ 15054-000 São José do Rio Preto - SP, Brasil
}

Recebido em 5/6/08; aceito em 13/10/08; publicado na web em 26/1/09

\begin{abstract}
ANTIOXIDANT ACTIVITY OF LEMON SEED EXTRACT (Citrus limon) ADDED TO SOYBEAN OIL IN ACCELERATED INCUBATOR-STORAGE TEST. The aim of this work was to evaluate antioxidant activity of lemon seeds added to soybean oil, submitted to accelerated incubator-storage test and to determine its synergistic effect with the synthetic antioxidant TBHQ. The treatments Control, TBHQ ( $50 \mathrm{mg} / \mathrm{kg}$ ), LSE (2,400 mg/kg Lemon Seed Extract), Mixture 1 (LSE + $50 \mathrm{mg} / \mathrm{kg}$ TBHQ) and Mixture $2\left(\mathrm{LSE}+25 \mathrm{mg} / \mathrm{kg}\right.$ TBHQ) were prepared and subjected to the accelerated incubator-storage test at $60^{\circ} \mathrm{C}$ for 12 days; samples were taken every 3 days and analyzed regarding peroxide value and conjugated dienes. The results showed that antioxidant activity of the tested treatments were: TBHQ $=$ Mixture $1=$ Mixture $2>$ LSE $>$ Control .
\end{abstract}

Keywords: citrus limon; antioxidant activity; accelerated incubator-storage test.

\section{INTRODUÇÃO}

O limão é uma fruta cítrica rica em vitaminas, principalmente vitamina C, fibras e potássio. ${ }^{1}$ É extensamente cultivado em pomares e viveiros, pois induz a maturação precoce das frutas, proporcionando melhores preços no início da safra. É utilizado para a fabricação de sucos naturais e concentrado, além de ser tecnologicamente usado como flavorizante em alimentos, devido ao óleo essencial presente em sua casca. ${ }^{2}$

O Brasil destaca-se como o segundo maior produtor de frutos cítricos e o maior exportador de sucos cítricos. A produção brasileira de limão está localizada no estado de São Paulo, sendo o primeiro produtor destes frutos, representando $81,3 \%$ da produção, seguido por Rio de Janeiro e Bahia com 3,9 e 2,7\%, respectivamente. ${ }^{3}$

Grande parte do resíduo sólido das sementes de limão é uma fonte inexplorada de óleo que pode alcançar $55 \%$ de rendimento. ${ }^{4}$ Este óleo pode ser aproveitado pelas indústrias alimentícia, farmacêutica e de cosméticos.

As frutas cítricas são conhecidas por conterem antioxidantes naturais no óleo, na polpa, na semente e na casca. Segundo Pereira, ${ }^{5}$ os resultados obtidos dos extratos metanólicos de sementes de frutas cítricas, como o limão, indicaram atividade antioxidante inibindo o processo oxidativo em $73,4 \%$.

Com o objetivo de retardar os processos oxidativos, responsáveis por alterações na cor, sabor, textura e valor nutricional, alguns compostos antioxidantes são adicionados aos alimentos. Estas substâncias podem ser naturais ou sintéticas e atuam de diversas maneiras: interrompendo a cadeia de reações oxidativas; cedendo um hidrogênio a um radical lipídico livre e assumindo a forma de radical estável diminuindo, assim, o número de radicais livres; reduzindo a velocidade da oxidação e prolongando-se o período de indução, que consiste no tempo que o material analisado necessita para começar a apresentar sinais detectáveis de oxidação. ${ }^{6,7}$

Os antioxidantes podem ser classificados de acordo com seu mecanismo de ação como primários ou secundários. Os antioxidantes primários são, por exemplo, compostos fenólicos, atuam interrom-

\footnotetext{
*e-mail: njorge@ibilce.unesp.br
}

pendo a cadeia da reação através da doação de elétrons ou hidrogênio aos radicais livres, convertendo-os em produtos termodinamicamente estáveis ou reagem com radicais livres, formando o complexo lipídioantioxidante que, por sua vez, pode reagir com outro radical livre. O átomo de hidrogênio ativo do antioxidante é seqüestrado pelos radicais livres $\mathrm{R}^{\bullet}$ e ROO` com maior facilidade que os hidrogênios alílicos das moléculas insaturadas. Assim, formam-se espécies inativas para a reação em cadeia e um radical relativamente estável $\left(\mathrm{A}^{*}\right)$ procedente do antioxidante. ${ }^{8}$

Já os antioxidantes secundários retardam a reação de autoxidação por diferentes mecanismos, que incluem complexação com metais, seqüestro de oxigênio, decomposição de hidroperóxidos para formar espécie não radical, absorção da radiação ultravioleta ou desativação de oxigênio singlete. ${ }^{9}$

Existem diversos métodos utilizados para a avaliação dos parâmetros da oxidação, dentre eles podem ser citados a determinação do índice de peróxidos, os ácidos graxos residuais não oxidados, os dienos conjugados, os compostos aldeídicos, os ácidos oxidados e os compostos voláteis. ${ }^{10}$

A determinação do índice de peróxidos representa a diferença entre a formação e a decomposição de peróxidos, e é expressa em miliequivalentes de oxigênio ativo por kg de matéria graxa. No decurso da sua decomposição produzem-se compostos de natureza muito diversa (aldeídos, cetonas, hidroxiácidos, hidrocarbonetos, polímeros), os quais são genericamente designados produtos secundários. ${ }^{11}$

Os dienos conjugados absorvem a $232 \mathrm{~nm}$. Os produtos secundários da sua oxidação, em particular as -dicetonas ou as cetonas insaturadas, apresentam um máximo de absorção a $272 \mathrm{~nm}$. Esta diferença é particularmente interessante, permitindo diferenciar estados de evolução oxidativa com base na relação $\mathrm{A}_{272 \mathrm{~nm}} / \mathrm{A}_{232 \mathrm{~nm}}$. Quanto maior o valor da absorbância a $232 \mathrm{~nm}$, mais elevado será o conteúdo em peróxidos, correspondendo, portanto, ao início do processo de oxidação; pelo contrário, quanto maior for o valor da absorbância a $272 \mathrm{~nm}$, maior será o teor de produtos secundários presentes. ${ }^{12-14}$

O emprego de antioxidantes sintéticos na indústria de alimentos tem sido alvo de questionamentos quanto à sua inocuidade e, devido a isso, pesquisas estão voltadas para a busca de compostos naturais que exibam esta propriedade funcional, atuando sozinhos 
ou sinergisticamente com outros aditivos, como alternativa para prevenir a deterioração oxidativa de alimentos e diminuir o uso dos antioxidantes sintéticos. ${ }^{15}$ Dentre as fontes de antioxidantes naturais podem ser citados os cereais, os cogumelos, as ervas e especiarias e as sementes de frutas cítricas. ${ }^{5,16-18}$

Diante disso, este trabalho objetivou a avaliação da atividade antioxidante do extrato metanólico de sementes de limão, adicionado ao óleo de soja submetido ao teste acelerado em estufa e, também, seu efeito sinergístico com o antioxidante sintético terc-butil-hidroquinona (TBHQ), utilizado atualmente pelas indústrias de alimentos com a finalidade de retardar as alterações oxidativas.

\section{PARTE EXPERIMENTAL}

\section{Sementes de limão}

As frutas maduras, variedade galego, foram provenientes de uma plantação na região de Itajobi-SP, colhidas em janeiro de 2007. Os limões foram cortados pela metade e as sementes retiradas manualmente e, em seguida, lavadas ligeiramente com água destilada para remover restos de polpas e açúcares solúveis provenientes das frutas. As sementes foram secas em estufa, com circulação de ar forçada, a $45^{\circ} \mathrm{C}$ por 24 h para redução do teor de umidade abaixo de $10 \%$. Depois, foram armazenadas em recipientes plásticos, vedados com tampas de rosca e devidamente rotulados, para análises posteriores.

\section{Óleo de soja}

Para a realização do experimento foi utilizado óleo de soja refinado, sem adição de antioxidantes sintéticos (TBHQ e ácido cítrico). Utilizou-se embalagens contendo $900 \mathrm{~mL}$ de óleo de soja, processado pela empresa Cargill Agrícola S/A, Uberlândia-MG.

\section{Antioxidantes}

$\mathrm{O}$ antioxidante sintético utilizado foi o terc-butil-hidroquinona (TBHQ), apresentado na forma em pó, fornecido pela empresa Danisco S/A.

O extrato metanólico de sementes de limão foi obtido de acordo com a metodologia descrita por Pereira. ${ }^{5}$ As sementes de limão desidratadas e trituradas $(10 \mathrm{~g})$ foram mantidas sob agitação permanente com metanol $(100 \mathrm{~mL})$ à temperatura ambiente $\left(25 \pm 2{ }^{\circ} \mathrm{C}\right)$, durante $6 \mathrm{~h}$ e, em seguida, a mistura foi centrifugada a $3.000 \mathrm{rpm}$, por $10 \mathrm{~min}$. Após a transferência do sobrenadante, o precipitado foi novamente submetido ao processo de extração nas mesmas condições anteriormente explicitadas, e os sobrenadantes resultantes da extração foram combinados. Em seguida, procedeu-se à remoção do solvente utilizado para a obtenção do extrato metanólico, sob pressão reduzida a $40{ }^{\circ} \mathrm{C}$. O extrato seco foi pesado e ressuspenso em metanol, obtendo-se solução-estoque contendo $1 \mathrm{~g}$ de extrato metanólico para cada $10 \mathrm{~g}$ de solvente metanol (1:10), utilizada para aplicação direta no óleo de soja.

\section{Ensaio experimental}

Foram submetidos ao teste de oxidação acelerada em estufa a $60{ }^{\circ} \mathrm{C}$ cinco tratamentos, conduzidos com duas repetições: óleo de soja, sem adição de antioxidantes sintéticos e ácido cítrico (Controle); óleo de soja, com adição de $2.400 \mathrm{mg} / \mathrm{kg}$ de extrato de sementes de limão (ESL); óleo de soja, com adição de $50 \mathrm{mg} / \mathrm{kg}$ de TBHQ (TBHQ); óleo de soja, com adição da mistura de antioxidantes, ou seja, $2.400 \mathrm{mg} / \mathrm{kg}$ de extrato de sementes de limão galego e $50 \mathrm{mg} / \mathrm{kg}$ de TBHQ (Mistura 1) e, óleo de soja, com adição da mistura de antioxidantes, ou seja, $2.400 \mathrm{mg} / \mathrm{kg}$ de extrato de sementes de limão galego e $25 \mathrm{mg} / \mathrm{kg}$ de TBHQ (Mistura 2).

Os tratamentos foram conduzidos por 12 dias em estufa aquecida, utilizando-se béqueres de $50 \mathrm{~mL}$ contendo $30 \mathrm{~mL}$ de amostra com relação superfície/volume $0,4 \mathrm{~cm}^{-1}$. Esse valor corresponde àquele normalmente usado nas frituras em fritadeira. A temperatura utilizada foi de $60{ }^{\circ} \mathrm{C}$, normalmente utilizada em testes de estocagem acelerada. ${ }^{19,20}$

Todas as amostras, a diferentes intervalos de tempo $(0,3,6,9 \mathrm{e}$ 12 dias), foram recolhidas e analisadas quanto ao índice de peróxidos e dienos conjugados.

\section{Métodos analíticos}

Com o objetivo de avaliar as alterações oxidativas primárias, utilizaram-se análises de peróxidos e dienos conjugados para avaliação das amostras obtidas durante o teste de estocagem acelerada.

\section{Índice de peróxidos}

Denomina-se índice de peróxidos a quantidade de oxigênio ativo, calculada em miliequivalentes, contida em $1 \mathrm{~kg}$ de óleo, medida a partir do iodo liberado do iodeto de potássio pelos peróxidos presentes no óleo. Esse índice foi determinado segundo a norma da American Oil Chemists' Society (AOCS) Cd 8-53. ${ }^{21}$

\section{Dienos conjugados}

Este método determina dienos conjugados presentes na matéria graxa, expressos como porcentagem de ácidos dienóicos conjugados, após leitura da absorbância a $233 \mathrm{~nm}$. A determinação de dienos conjugados foi efetuada de acordo com o método oficial AOCS Ti 1a-64. ${ }^{21}$

\section{Delineamento experimental}

Para as amostras submetidas ao teste acelerado em estufa foram considerados os seguintes fatores: tratamentos (Controle, ESL, TBHQ, Mistura 1 e Mistura 2) e tempos de aquecimento (0, 3, 6, 9 e 12 dias).

Os resultados obtidos para índice de peróxidos e dienos conjugados, em duas repetições, foram submetidos às análises de variância para determinar a influência dos fatores sobre a alteração dos óleos submetidos ao teste acelerado em estufa. $\mathrm{O}$ experimento foi realizado em esquema fatorial 5 x 5 , no delineamento inteiramente casualizado. ${ }^{22} \mathrm{~A}$ análise de variância e o teste de Tukey para as médias a 5\% foram obtidos por meio do programa ESTAT - Sistema para Análises Estatísticas, versão 2.0. ${ }^{23}$

\section{RESULTADOS E DISCUSSÃO}

\section{Índice de peróxidos}

O índice de peróxidos representa a diferença entre a formação e a decomposição de peróxidos; produtos primários de oxidação, incolores e inodoros. Os peróxidos são intermediários instáveis, sobretudo a temperaturas elevadas. ${ }^{10}$

A Tabela 1 apresenta os resultados médios de índice de peróxidos para os tratamentos Controle, ESL, TBHQ, Mistura 1 e Mistura 2, submetidos ao teste acelerado em estufa a $60{ }^{\circ} \mathrm{C}$ por 12 dias. Para cada tratamento verificou-se que os valores aumentaram ao longo do período de estocagem, havendo diferença significativa entre os tempos de aquecimento.

Analisando os tratamentos dentro de cada tempo de aquecimento, nota-se que até 3 dias não foi observada diferença significativa entre 
Tabela 1. Médias do índice de peróxidos (meq/kg) do óleo de soja controle e do óleo de soja com adição de antioxidantes para cada tratamento e tempo de aquecimento

\begin{tabular}{lccccc}
\hline \multirow{2}{*}{ Tratamentos } & \multicolumn{5}{c}{ Tempos de aquecimento (dias) } \\
\cline { 2 - 6 } & 0 & 3 & 6 & 9 & 12 \\
\hline Controle & $1,79^{\mathrm{eA}}$ & $2,89^{\mathrm{dA}}$ & $30,38^{\mathrm{cA}}$ & $57,09^{\mathrm{bA}}$ & $77,91^{\mathrm{aA}}$ \\
ESL & $1,60^{\mathrm{eA}}$ & $2,83^{\mathrm{dA}}$ & $10,54^{\mathrm{cB}}$ & $36,97^{\mathrm{bB}}$ & $57,65^{\mathrm{aB}}$ \\
TBHQ & $1,39^{\mathrm{eA}}$ & $2,84^{\mathrm{dA}}$ & $4,66^{\mathrm{cC}}$ & $7,66^{\mathrm{bC}}$ & $9,54^{\mathrm{aC}}$ \\
Mistura 1 & $1,64^{\mathrm{dA}}$ & $2,43^{\mathrm{dA}}$ & $3,87^{\mathrm{cC}}$ & $7,16^{\mathrm{bC}}$ & $9,31^{\mathrm{aC}}$ \\
Mistura 2 & $1,53^{\mathrm{eA}}$ & $2,69^{\mathrm{dA}}$ & $4,13^{\mathrm{cC}}$ & $7,34^{\mathrm{bC}}$ & $9,54^{\mathrm{aC}}$ \\
\hline
\end{tabular}

Controle: óleo de soja; ESL: extrato de sementes de limão (2.400 mg/ $\mathrm{kg}$ ); TBHQ: terc-butil-hidroquinona $(50 \mathrm{mg} / \mathrm{kg})$; Mistura 1: extrato de sementes de limão (2.400 mg/kg) + TBHQ (50 mg/kg); Mistura2: extrato de sementes de limão $(2.400 \mathrm{mg} / \mathrm{kg})+$ TBHQ $(25 \mathrm{mg} / \mathrm{kg})$. a, b... (linha): em cada Tratamento, médias de Tempos de aquecimento seguidas de mesma letra minúscula não diferem pelo teste de Tukey (P > 0,05). A, B... (coluna): em cada Tempo de aquecimento, médias de Tratamentos seguidas de mesma letra maiúscula não diferem pelo teste de Tukey $(\mathrm{P}>0,05)$.

as médias dos índices de peróxidos. A partir de 6 dias de aquecimento, os teores de peróxidos do óleo de soja com TBHQ, Mistura 1 e Mistura 2 não diferiram significativamente, apresentando-se mais eficientes na proteção do óleo, com relação à formação de peróxidos. O extrato de sementes de limão reduziu em $26 \%$, o TBHQ e as Misturas 1 e 2 reduziram em $88 \%$ a formação de peróxidos do óleo após 12 dias de estocagem acelerada a $60^{\circ} \mathrm{C}$. Nota-se que a adição do extrato metanólico de sementes de limão ao óleo de soja não foi tão eficiente quanto o antioxidante sintético TBHQ e as misturas de antioxidantes, contra a formação de peróxidos.

Com relação à eficiência contra a formação dos compostos primários de oxidação durante o período de estocagem em estufa, pode-se classificar os tratamentos da seguinte maneira: TBHQ $=$ Mistura $1=$ Mistura 2> ESL > Controle.

O TBHQ tem se mostrado mais eficiente que os antioxidantes naturais, quando adicionado na dosagem máxima permitida de 200 $\mathrm{mg} / \mathrm{kg}$. Gámez-Meza et al. ${ }^{24}$ verificaram que o TBHQ obteve melhor resultado que o extrato de bagaço de uvas, contra a formação de peróxidos, em óleo de soja, durante estocagem a $60{ }^{\circ} \mathrm{C}$.

Almeida-Doria e Regitano-D’ Arce ${ }^{19}$ verificaram que o TBHQ, aplicado na concentração de $200 \mathrm{mg} / \mathrm{kg}$, foi mais eficiente na proteção contra a formação de peróxidos em óleo de soja que extratos etanólicos de alecrim e orégano, a partir de 5 e 7 dias em teste de estocagem acelerada a $63{ }^{\circ} \mathrm{C}$.

Em estudo realizado por Andreo e Jorge, ${ }^{25}$ observou-se que o extrato de gengibre, TBHQ e a mistura, antioxidante sintético TBHQ com o extrato etanólico de gengibre, adicionados no óleo de soja reduziram em 57, 90 e $92 \%$, respectivamente, a formação de peróxidos após 12 dias de estocagem acelerada a $60{ }^{\circ} \mathrm{C}$.

Rehman ${ }^{26}$ verificou que o extrato de cascas de citros adicionado em óleo de milho, na concentração de 2.000 ppm, apresentou efeito semelhante ao do antioxidante sintético BHT (200 ppm) na proteção contra a formação de peróxidos em teste de estocagem acelerada a $45^{\circ} \mathrm{C}$ por 6 meses.

\section{Dienos conjugados}

A medida quantitativa dos dienos conjugados tem sido largamente utilizada para a determinação da oxidação de óleos e gorduras. A peroxidação dos ácidos graxos insaturados acompanha a mudança da dupla ligação na formação dos hidroperóxidos conjugados. Essa estrutura conjugada absorve fortemente a luz ultravioleta no comprimento de onda entre 232 e $234 \mathrm{~nm}^{27}$

De acordo com a Tabela 2, houve aumento gradual na formação de dienos conjugados ao longo do teste acelerado em estufa, de forma progressiva, do início ao final do aquecimento tanto para o óleo de soja controle quanto para o óleo de soja com adição dos antioxidantes testados.

Tabela 2. Médias de dienos conjugados (\%) do óleo de soja controle e do óleo de soja com adição de antioxidantes para cada tratamento e tempo de aquecimento

\begin{tabular}{lccccc}
\hline \multirow{2}{*}{ Tratamentos } & \multicolumn{5}{c}{ Tempos de aquecimento (dias) } \\
\cline { 2 - 6 } & 0 & 3 & 6 & 9 & 12 \\
\hline Controle & $0,25^{\mathrm{eA}}$ & $0,28^{\mathrm{dA}}$ & $0,59^{\mathrm{cA}}$ & $0,92^{\mathrm{bA}}$ & $1,14^{\mathrm{aA}}$ \\
ESL & $0,24^{\mathrm{eA}}$ & $0,28^{\mathrm{dA}}$ & $0,53^{\mathrm{cB}}$ & $0,85^{\mathrm{bB}}$ & $1,06^{\mathrm{aB}}$ \\
TBHQ & $0,24^{\mathrm{eA}}$ & $0,27^{\mathrm{dA}}$ & $0,39^{\mathrm{cC}}$ & $0,49^{\mathrm{bC}}$ & $0,57^{\mathrm{aC}}$ \\
Mistura 1 & $0,25^{\mathrm{dA}}$ & $0,27^{\mathrm{dA}}$ & $0,39^{\mathrm{cC}}$ & $0,50^{\mathrm{bC}}$ & $0,58^{\mathrm{aC}}$ \\
Mistura 2 & $0,24^{\mathrm{eA}}$ & $0,26^{\mathrm{dA}}$ & $0,38^{\mathrm{cC}}$ & $0,50^{\mathrm{bC}}$ & $0,58^{\mathrm{aC}}$ \\
\hline
\end{tabular}

Controle: óleo de soja; ESL: extrato de sementes de limão (2.400 mg/ $\mathrm{kg}$ ); TBHQ: terc-butil-hidroquinona (50 mg/kg); Mistura 1: extrato de sementes de limão (2.400 mg/kg) + TBHQ (50 mg/kg); Mistura2: extrato de sementes de limão $(2.400 \mathrm{mg} / \mathrm{kg})+$ TBHQ $(25 \mathrm{mg} / \mathrm{kg})$. a, b... (linha): em cada Tratamento, médias de Tempos de aquecimento seguidas de mesma letra minúscula não diferem pelo teste de Tukey (P>0,05). A, B... (coluna): em cada Tempo de aquecimento, médias de Tratamentos seguidas de mesma letra maiúscula não diferem pelo teste de Tukey $(\mathrm{P}>0,05)$.

Pode-se observar, portanto, que até o tempo de aquecimento de 3 dias não houve formação significativa de dienos conjugados nos tratamentos utilizados. Esta formação começou a ser detectada estatisticamente apenas a partir do tempo de aquecimento de 6 dias em estufa. A partir daí, observa-se que os tratamentos ESL, TBHQ, Mistura 1 e Mistura 2 foram eficientes contra a formação de dienos conjugados, pois diferiram do controle. A maior formação de dienos no óleo indica formação de compostos primários de oxidação lipídica mais acentuada nesse óleo que nos demais tratamentos.

Ao final do processo, pode-se verificar que o extrato de sementes de limão demonstrou efeito antioxidante durante todo o aquecimento, embora tenha apresentado o menor nível de proteção, com $7 \%$ de redução da formação de dienos conjugados após 12 dias de estocagem acelerada. A aplicação do antioxidante sintético TBHQ e das misturas de TBHQ e extrato de sementes de limão foram mais efetivas entre os tratamentos testados, embora não tenham apresentado diferença significativa entre si na proteção do óleo de soja refinado estocado a $60{ }^{\circ} \mathrm{C}$ com relação à formação de dienos conjugados. O óleo adicionado de TBHQ apresentou redução de 50\%, e as misturas de TBHQ e extratos de sementes de limão apresentaram $49 \%$ de redução na formação desses compostos no final do processo.

Os extratos de frutas cítricas como o limão são importantes fontes de ácidos fenólicos, como o ácido hidroxicinâmico e de flavonóides (flavononas e flavonóis).$^{28}$ Esses compostos podem agir como redutores, interruptores de radicais livres, inibidores ou supressores de oxigênio singlete e como inativadores de metais pró-oxidantes.

Ruth, Shaker e Morrissey ${ }^{29}$ observaram redução de $23 \%$ no valor de dienos conjugados em óleo de linhaça, com a adição de $4 \%$ de extrato metanólico de semente de soja. O extrato etanólico de manjericão retardou a formação de dienos conjugados durante a estocagem 
de carne de porco, evidenciando a utilização de extratos naturais com características polares contra a oxidação lipídica. ${ }^{30}$

A comparação entre antioxidantes sintéticos e naturais quanto à capacidade antioxidante depende do tipo do composto analisado e da concentração utilizada. Os valores de dienos conjugados em óleo de milho com adição de $20 \%$ de extrato etanólico de germe de trigo torrado foram inferiores ao do óleo contendo 0,02\% de BHA, em estudo realizado por Krings et al. ${ }^{31}$

Iqbal e Bhanger ${ }^{32}$ estudaram a formação de dienos conjugados em óleo de girassol adicionado de extrato metanólico de alho, em estocagem acelerada. As concentrações de 250, 500 e $1.000 \mathrm{mg} / \mathrm{kg}$ foram comparadas com os antioxidantes sintéticos BHA e BHT (200 $\mathrm{mg} / \mathrm{kg}$ ). Após $80 \mathrm{~min}$ de aquecimento a $185^{\circ} \mathrm{C}$, concluíram que o óleo de girassol adicionado de $200 \mathrm{mg} / \mathrm{kg}$ de BHT foi o mais eficiente na inibição da formação de dienos conjugados, seguido da amostra contendo $1.000 \mathrm{mg} / \mathrm{kg}$ de extrato metanólico de alho.

Em estudo realizado por Angelo e Jorge, ${ }^{33}$ extrato de coentro, palmitato de ascorbila e mistura destes antioxidantes quando adicionados no óleo de girassol apresentaram capacidade em retardar a formação de dienos conjugados em 11,2, 56,9 e 60,9\%, respectivamente, após 10 dias de estocagem acelerada.

Contudo, ao final do teste acelerado em estufa, ou seja, aos 12 dias de aquecimento, os valores resultantes das determinações de índice de peróxidos e dienos conjugados apresentaram coeficiente de correlação elevado e positivo $(\mathrm{r}=0,9924)$ para as médias dos tratamentos utilizados.

\section{CONCLUSÃO}

Apesar de o antioxidante sintético TBHQ ter apresentado proteção superior ao controle, na concentração utilizada neste estudo, deve-se ressaltar que a Mistura 2 teve comportamento semelhante, sendo capaz de prevenir a oxidação lipídica. Portanto, recomenda-se às indústrias de óleos vegetais diminuir a concentração do antioxidante sintético, TBHQ, substituindo-o parcialmente pelo antioxidante natural.

A atividade antioxidante dos diversos tratamentos testados neste estudo foi: $\mathrm{TBHQ}=$ Mistura $1=$ Mistura $2>\mathrm{ESL}>$ Controle .

\section{AGRADECIMENTOS}

À Coordenação de Aperfeiçoamento de Pessoal de Nível Superior - CAPES, pela concessão da bolsa de mestrado e ao Conselho Nacional de Desenvolvimento Científico e Tecnológico - CNPq, pela bolsa de Produtividade em Pesquisa.

\section{REFERÊNCIAS}

1. Ferrari, C. K. B.; Torres, E. A. F. S.; Rev. Bras. Cancerol. 2002, 48, 382.

2. Reda, S. Y.; Leal, E. S.; Batista, E.; Barana, A. C.; Schnitzels, E.; Carneiro, P. I. B.; Cienc. Tecnol. Aliment. 2005, 25, 676.

3. http://www.ibraf.org.br, acessada em Maio 2008.

4. Fernandes, J. B.; David, V.; Facchini, P. H.; da Silva, M. F. das G. F.; Rodrigues Filho, E.; Vieira, P. C.; Galhiane, M. S.; Pagnocca, F. C.; Bueno, O. C.; Hebling, M. J.; Victor, S. R.; dos Santos, A. M. R.; Quim. Nova 2002, 25, 1091.
5. Pereira, R. B.; Dissertação de Mestrado, Universidade de São Paulo, Brasil, 1996.

6. Amarowicz, R.; Pegg, R. B.; Rahimi-Moghaddam, P.; Barl, B.; Weil, J. A.; Food Chem. 2004, 84, 562.

7. Ordóñez, J. A.; Rodríguez, M. I. C.; Alvarez, L. F.; Sanz, M. L. G.; Minguillón, G. D. G. F.; Perales, L. I. H.; Cortecero, M. D. S.; Tecnologia de alimentos: componentes dos alimentos e processos, Artmed: Porto Alegre, 2005, vol. 1.

8. Ramalho, V. C.; Jorge, N.; Quim. Nova 2006, 29, 755.

9. Gordon, M. H. Em The mechanism of antioxidant action in vitro; Hudson, B. J. F., ed.; Elsevier Applied Science: London, 1990, cap. 1.

10. Silva, F. A. M.; Borges, M. F. M.; Ferreira, M. A.; Quim. Nova 1999, 22, 103 .

11. Berset, C.; Cuvelier, M.-E.; Sciences des Aliments 1996, 16, 219.

12. Frankel, E. N.; Huang, S.-W.; Kanner, J.; German, J. B.; J. Agric. Food Chem. 1994, 42, 1054.

13. Huang, S.-W.; Hopia, A.; Schwarz, K.; Frankel, E. N.; German, J. B.; J. Agric. Food Chem. 1996, 44, 444.

14. Srinivasan, S.; Xiong, Y. L.; Decker, E. A.; J. Agric. Food Chem. 1996, 44, 119.

15. Pokorný, J.; Eur. J. Lipid. Sci. Tech. 2007, 109, 642.

16. Elmastas, M.; Isildak, O.; Turkekul, I.; Temur, N.; J. Food Compos. Anal. 2007, 20, 345.

17. Pérez-Jiménez, J.; Saura-Calixto, F.; J. Agric. Food Chem. 2005, 53, 5040.

18. Yanishlieva, N. V.; Marinova, E.; Pokorný, J.; Eur. J. Lipid. Sci. Tech. 2006, 108, 793.

19. Almeida-Doria, R. F.; Regitano-D'arce, M. A. B.; Cienc. Tecnol. Aliment. 2000, 20, 14 .

20. Kim, S. Y.; Jeong, S. M.; Park, W. P.; Nam, K. C.; Ahn, D. U.; Lee, S. C.; Food Chem. 2005, 97, 479.

21. AOCS - American Oil Chemists Society; Official methods and recommended practices of the American Oil Chemists' Society, Champaign: AOCS, 1993.

22. Banzatto, D. A.; Kronka, S. N.; Experimentação agrícola, Funep: Jaboticabal, 2006.

23. Unesp; ESTAT - Sistema para Análises Estatísticas, Jaboticabal, 1999, Versão 2.0. 1 disquete.

24. Gámez-Meza, N.; Noriega-Rodríguez, J. A.; Medina-Juárez, L. A.; Ortega-García, J.; Cázarez-Casanova, R.; Angulo-Guerrero, O.; J. Am. Oil Chem. Soc. 1999, 76, 1447.

25. Andreo, D.; Jorge, N.; Rev. Inst. Adolfo Lutz 2007, 66, 157.

26. Rehman, Z.; Food Chem. 2006, 99, 454.

27. Kulas, E.; Ackman, R.; J. Agric. Food Chem. 2001, 49, 1729.

28. Dimitrios, B.; Trends Food Sci. Technol. 2006, 17, 505.

29. Ruth, S. M. V.; Shaker, E. S.; Monrrissey, P. A.; Food Chem. 2001, 75, 184.

30. Juntachote, T.; Berghofer, E.; Siebenhandl, S.; Bauer, F.; Food Chem. 2007, 100, 129.

31. Krings, U.; El-Saharty, Y. S.; El-Zeany, B. A.; Pabel, B.; Berger, R. G.; Food Chem. 2000, 71, 95.

32. Iqbal, S.; Bhanger, M. I.; Food Chem. 2007, 100, 254.

33. Angelo, P. M.; Jorge, N.; Cienc. Tecnol. Aliment. 2008, 28, 79. 